\title{
CONTROLLABILITY ANALYSIS OF AN UNSTABLE, NON-MINIMUM PHASE PROCESS
}

\author{
Espen Storkaas* Sigurd Skogestad ${ }^{*, 1}$ \\ * Department of Chemical Engineering, Norwegian \\ University of Science and Technology, Trondheim, Norway
}

\begin{abstract}
The choice of manipulated inputs and measured outputs can be critical for the successful design of a control system. This is especially the case when designing stabilizing controllers for unstable, non-minimum phase systems, as unstable poles combined with unstable zeros and time delay can render stabilizing control very hard. Since the presence and location of unstable zeros and time delay usually depends on the choice of measurements and control actuators, a thorough analysis prior to the actual controller design is important. In this paper, a controllability analysis is performed on a pipeline-riser system with multiphase flow, where the design objective is to stabilize an unstable operating point to remove riser slugging. Copyright (C) 2005 IFAC
\end{abstract}

Keywords: Controllability, Stabilizing Control, Unstable, Non-minimum phase, Feedback control

\section{INTRODUCTION}

When designing control systems, there are a number of decisions to be made prior to the actual design of the controllers. These decisions include what variables to measure, which variables to manipulate and how the manipulated and measured variables should be paired. In addition, information as to whether acceptable performance is possible without redesigning the process would be valuable at an early stage in a design process. These questions are especially important when designing stabilizing controllers for unstable, nonminimum phase systems, where the choice of measurements and manipulated variables can be the deciding factor for a successful design.

A controllability analysis is an investigation into a system's fundamental performance limitations with respect to control. Skogestad and Postleth-

\footnotetext{
1 Author to whom correspondence should be addressed: skoge@chemeng.ntnu.no
}

waite (1996) defines controllability as a systems ability to achieve acceptable control performance; that is to keep the systems outputs $(y)$ within specified bounds or displacement from the references $(r)$, in spite of unknown but bounded variations, such as disturbances (d) and plant changes, using available inputs $(u)$ and available measurements $\left(y_{m}\right.$ or $\left.d_{m}\right)$. By performing a controllability analysis for the system with all the possible candidates for measurements and manipulative variables, the set of variables that results in the most controllable system can be chosen.

In order to illustrate the importance of a controllability analysis, an example from the offshore oil industry is studied. Multiphase flow of oil and gas in pipeline-riser system can give rise to an oscillatory flow regime called riser slugging. Riser slugging can be removed by stabilizing an unstable operating point existing at the same boundary conditions using feedback control. The pipelineriser system is very interesting, as it is an unstable 
system which, depending on the measured input, contains both unstable zeros and time delay. In addition, the unstable poles constitute a complex pair, something which adds additional complexity to the analysis.

The pipeline-riser system is described in section 2 . In section 3, the system-theoretical background for the controllability analysis is given, and in section 4 , the results from the controllability analysis is discussed.

\section{SYSTEM DESCRIPTION}

\subsection{Riser Slugging Phenomenon}

Most of the newly discovered, smaller oil fields on the Norwegian continental shelf are being tied in to existing infrastructure by transporting the untreated wellstream in multiphase transport pipelines from the wellhead clusters to the production platform. Riser slugging is a flow pattern in multiphase flow characterized by varying or irregular flows and surges of liquid and gas in the pipeline-riser system. Riser slugging is caused by liquid accumulation in the bottom of the riser, which blocks the pipeline cross-section and forms a liquid slug. The slug grows both up into the riser and backwards into the pipeline as more liquid is added, whilst the gas is trapped upstreams of the slug causing increased pressure. When the pressure drop over the riser overcomes the hydrostatic head of the liquid slug, the liquid is transported out of the riser followed by a burst of gas. After the gas production, the slug cycle starts over. This cyclic behavior is illustrated in figure 1. More information on riser slugging can be found in Taitel (1986).

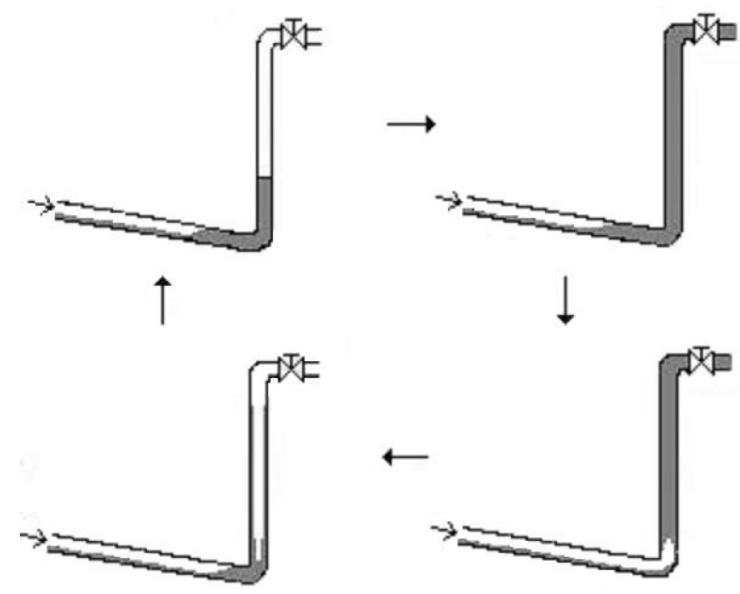

Fig. 1. Graphic illustration of a riser slugging cycle

The highly irregular flow out of the pipeline caused by riser slugging may lead to severe problems for the receiving facility. Among these prob- lems are; the large amounts of liquid in the slug can cause separator overfilling and process trip and in less severe cases poor separation and the irregular gas production causes varying compressor loads and unnecessary flaring. Hence, riser slugging must be avoided in pipeline-riser systems.

Recently, feedback control has emerged as the preferred solution for avoiding riser slugging in pipeline-riser systems. Controller design, experiments and industrial implementations has been reported (Schmidt et al., 1979; Hedne and Linga, 1990; Hollenberg et al., 1995; Courbot, 1996; Henriot et al., 1999; Havre et al., 2000; Havre and Dalsmo, 2002; Kovalev et al., 2003; Skofteland and Godhavn, 2003; Storkaas et al., 2003). These control systems are designed either to keep the system away from the riser slugging conditions or to stabilize the unstable operating point existing at the same boundary conditions as riser slugging.

\subsection{Case Description}

In order to study the dominant dynamic behavior of a typical, yet simple severe slugging problem, the test case for severe slugging in OLGA is used. OLGA is a commercial multiphase simulator widely used in the oil industry. The nomenclature used for these systems are given in figure 2(a). The case geometry is given in figure $2(b)$. The pipe diameter is $0.12 \mathrm{~m}$. The feed into the system is constant at $9 \mathrm{~kg} / \mathrm{s}$, with $W_{L}=8.64 \mathrm{~kg} / \mathrm{s}$ oil and $W_{G}=0.36 \mathrm{~kg} / \mathrm{s}$ gas. The pressure behind the choke valve $\left(P_{0}\right)$ is constant at 50 bar. This leaves the choke valve opening as the only degree of freedom in the system.

The stability of the flow is dependent on the topside choke valve opening $Z$. It is usually possible to obtain stable flow (that is, no riser slugging) by a sufficiently low opening $Z$. This is illustrated in figure 2.2 , were the pressure at the inlet $P_{I}$ is show for simulations with choke valve openings $Z=10$, 20 and $40 \%$.

To illustrate behavior of the system over the whole working range of the choke valve, the bifurcation diagrams in figure 4 are useful. The thickest lines represent the OLGA reference data. The solid lines show the observed pressure as a function of valve opening. For small valve openings, the flow in the system is stable and the inlet pressure $P_{I}$ in figure $4(\mathrm{a})$ and the pressure drop over the topside choke valve $D P$ in figure 4(b) are constant as shown by single solid lines. For large valve openings there is riser slugging in the system, indicated in the bifurcation diagrams by plotting both the maximum and minimum pressure in the oscillations. In other words, $P_{I}$ and $D P$ oscillates between the upper and lower solid lines. The 


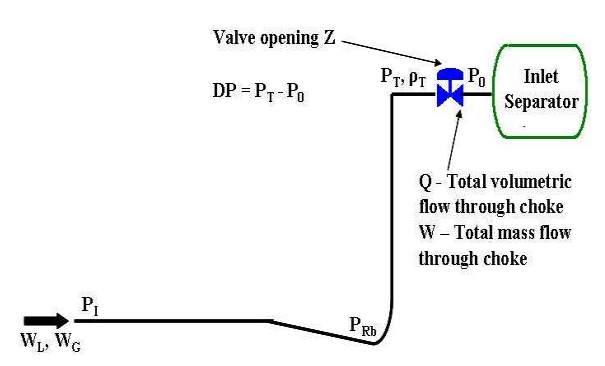

(a) Nomenclature

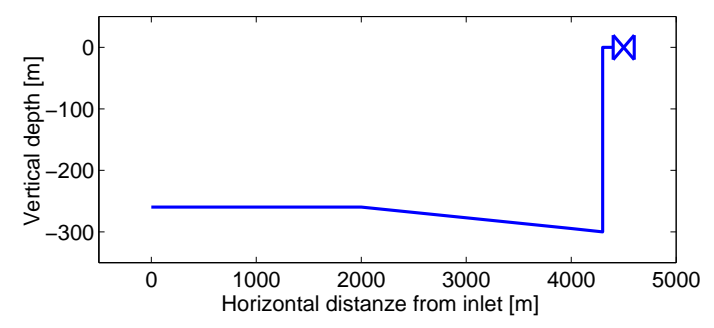

(b) Case Geometry

Fig. 2. (a) Nomenclature used for the pipeline riser system and (b) System geometry
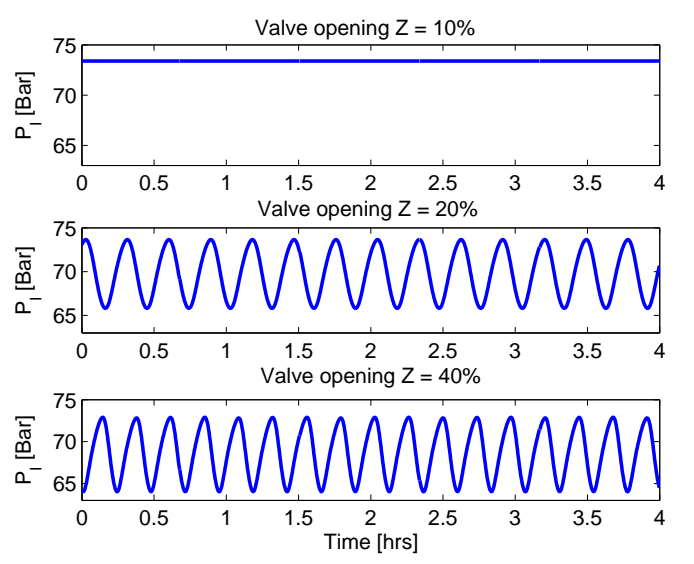

Fig. 3. OLGA simulations for valve openings of $Z=10,20$ and $40 \%$

dashed line represents the unstable steady-state solution. This is our desired operating line with closed-loop operation.

\subsection{Model tuning}

As multiphase flow in pipelines is very complex and the models describing it are complicated, two different simplified models are used to describe the system. The assumption is that if these two models predict the same qualitative controllability properties, the results should be reasonably robust toward modeling error. The first model is a PDEbased two-fluid model (Storkaas et al., 2001), the other a simplified model with three dynamical states tailor-made for controller design and analysis (Storkaas et al., 2003).

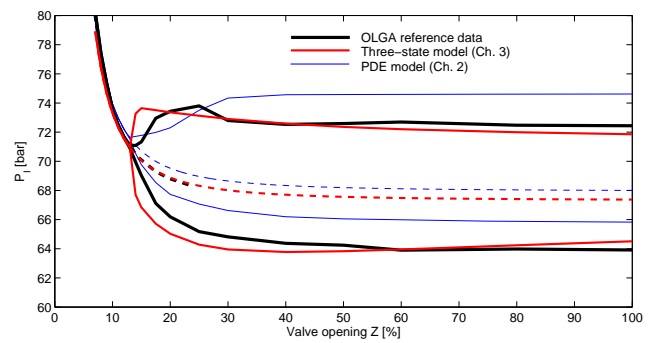

(a) Inlet Pressure

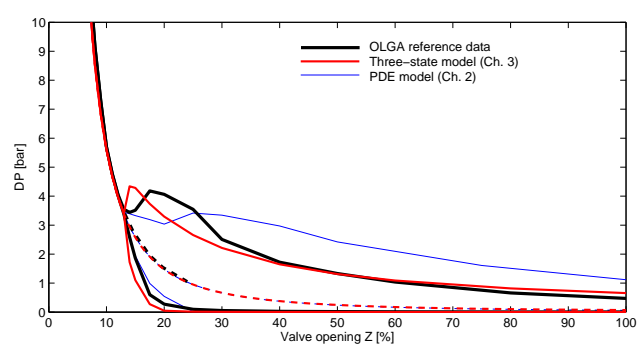

(b) Pressure drop over choke

Fig. 4. Model tuning and verification for (a) Inlet pressure $P_{I}$ and (b) Pressure drop over choke valve $D P$

The two models are fitted to the reference data, and the resulting responses are shown in figure 4. The figure shows that both models have a correct qualitative behavior, but the pressure drop over the pipeline (as represented by the inlet pressure $P_{I}$ ) for the PDE-based model is about 0.7 bar too high. There are some deviations for the riser slugging amplitude for both models, but this is of lesser importance as the control system should stay away from this solution.

\section{CONTROLLABILITY THEORY}

\subsection{Limitations imposed by unstable zeros}

The presence of unstable (RHP) zeros $z$ may cause severe problems for stabilizing control. Youla et al. (1974) prove that for a strictly proper SISO plant $G(s)$ to be stabilized by a stable controller, every real RHP-zero in $G(s)$ must lie to the left of an even number of real RHP-poles in $G(s)$. For pairs of complex conjugate unstable poles, the result from Youla et al. (1974) does not fundamentally limit the possibility for a stabilizing controller to be found, irrespective of the possible presence of RHP-zeros.

Skogestad and Postlethwaite (1996) show that achievable performance is closely linked to the distance between unstable poles and unstable zeros. For real poles, they derive the approximate performance requirement $z>4 p$ based on demanding the maximum peak of the complementary sensitivity function $T$ to be less than 2 , $M_{T}<2$. Skogestad and Postlethwaite (1996) also 
show that complex unstable poles and zeros are more troublesome the closer they are to the real axis relative to the imaginary axis.

\subsection{Lower bounds on sensitivity peaks}

The distance between the unstable poles and zeros is closely related to the lowest achievable peaks in sensitivity and complementary functions, denoted $M_{S, \min }$ and $M_{T, \min }$, respectively. Based on the work of Chen (2000), the values for $M_{S, \min }$ and $M_{T, \text { min }}$ can be computed. $M_{S, \text { min }}$ and $M_{T, \text { min }}$ are good indications of the theoretically achievable performance for a system, but to actually achieve the computed peak values may require very complicated controllers. Note that the value for these bounds only depends on the relative location of the unstable poles and zeros.

To avoid excessive input usage and input saturation, the transfer function $K S$ from measurement noise $n$ to plant input $u$ should small. By small we mean that for a properly scaled system, the maximum amplification of the signal through the transfer function $K S$ minimized over all possible controllers $K, \min _{K}\|K S(s)\|_{\infty}$ should be less than one in magnitude. Based on the work of Francis (1987), an exact value of this norm can be calculated using equation 1

$$
\min _{K}\|K S(s)\|_{\infty}=\frac{1}{\underline{\sigma}_{H, u}(G(s))}
$$

If the bound in equation 1 is less then unity for a properly scaled system, the plant can theoretically be stabilized using the available inputs.

\section{CONTROLLABILITY ANALYSIS}

The models are linearized around two different unstable operating points, one "easy" (slow instability) corresponding to a valve opening of $17.5 \%$ and one "difficult" (faster instability) corresponding to a valve opening of $30 \%$. The PDE model results in a relatively large linear model (many states), as it even with a rather coarse discretization results in a set of 50 ODE's. The resulting linear models are scaled as outlined in Skogestad and Postlethwaite (1996).

\subsection{Stability - Poles}

When the valve opening is increased, the stationary operating point moves along the single solid line in figure 4 , through the bifurcation point at valve opening $Z=13 \%$ and onwards along the dashed line for the unstable operating points. At the bifurcation point, there is a pair of poles (eigenvalues of the state feedback matrix of the linearized model) that move into the right half plane as seen from the root-locus plot in figure 5 . This indicates that the bifurcation point is a Hopf bifurcation (Thompson and Stewart, 1986), which is also consistent with the shape of the bifurcation maps in figure 4

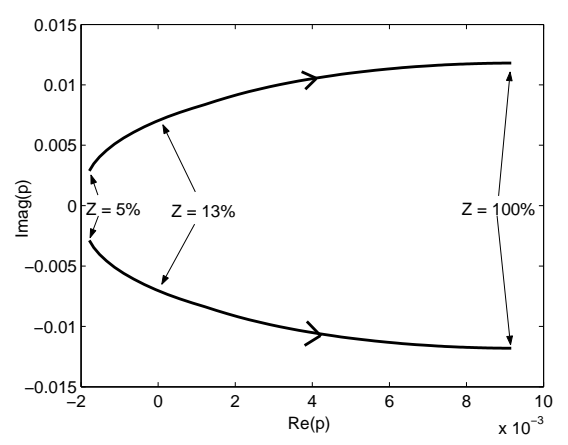

Fig. 5. Controllability analysis: Open-loop RootLocus plot with valve opening $Z$ as independent parameter. The results are computed using the PDE-based two-fluid model

\subsection{Poles and zeros - Measurement selection}

In this work we have considered the following measurement candidates: inlet pressure $P_{I}$, pressure drop over choke valve $D P$ and volumetric flow through the topside choke valve $Q$. Tables 1 and 2 give the location of the smallest RHPzero, the lower bound on the peaks of the sensitivity function $S$ and $T\left(M_{S, \min }=M_{T, \min }\right)$ and the lower bound on the sensitivity function $K S\left(\min _{K}\|K S\|_{\infty}\right)$ for these measurement candidates. The stationary process gain $|G(0)|$ is also included in the analysis to identify measurement candidates that has small low-frequency gain.

Tables 1 and 2 shows that it is theoretically possible to stabilize the system with all the measurement candidates as $\min _{K}\|K S\|_{\infty}<1$ in all cases. However, the unstable zero dynamics (RHP-zeros) associated with $y=D P$ makes it practically impossible to design an effective stabilizing controller using this measurement. Thus, the pressure drop over the topside choke valve $(y=D P)$ will be excluded from the following analysis. The controllability results from the two different models are similar, the differences can all be explained by the complexity difference between the two models.

4.2.1. Inlet pressure $P_{I} \quad$ The inlet pressure $P_{I}$ seems to be ideally suited as a measurement for stabilizing control of these systems as it has small lower bounds for all the sensitivity functions, no unstable (RHP) zeros and high stationary gain. This is reassuring since pressure control using the 
Table 1. Controllability data for the operating point $Z=17.5 \%$, unstable poles at $\omega=0.0007 \pm 0.0073$ for simplified model and $\omega=0.0014 \pm 0.0085$ for PDE-based two-fluid model.

\begin{tabular}{cccccc}
\hline Measurement & Value & Smallest RHP-zero & $\begin{array}{c}M_{S, \text { min }} \\
M_{T, \text { min }}\end{array}$ & $\min _{K}\|K S\|_{\infty}$ & $|G(0)|$ \\
\hline$P_{I}[$ bar $]$ (simp) & 69.35 & - & 1 & 0.01 & 19 \\
$P_{I}[$ bar $]$ (PDE) & 70 & 99 & 1.0 & 0.03 & 18.9 \\
\hline$D P[$ bar $]$ (simp) & 1.91 & 0.018 & 1.1 & 0.02 & 17.7 \\
$D P[$ bar $]$ (PDE) & 1.92 & $0.01 \pm 0.01 i$ & 1.6 & 0.04 & 17.6 \\
\hline$Q\left[\mathrm{~m}^{3} / \mathrm{s}\right](\mathrm{simp})$ & 0.0194 & - & 1 & 0.01 & 1.5 \\
$Q\left[\mathrm{~m}^{3} / \mathrm{s}\right](\mathrm{PDE})$ & 0.0208 & - & 1 & 0.02 & 1.8 \\
\hline
\end{tabular}

Table 2. Controllability data for the operating point $Z=30 \%$, unstable poles at $\omega=0.0038 \pm 0.0115 i$ for simplified model and $\omega=0.0045 \pm 0.0108$ for PDE-based two-fluid model.

\begin{tabular}{cccccc}
\hline Measurement & Value & Smallest RHP-zero & $\begin{array}{c}M_{S, \text { min }} \\
M_{T, \text { min }}\end{array}$ & $\min _{K}\|K S\|_{\infty}$ & $|G(0)|$ \\
\hline$P_{I}[$ bar $]$ (simp) & 68 & - & 1 & 0.11 & 3.4 \\
$P_{I}[$ bar $]$ (PDE) & 68.7 & 98.1 & 1.0 & 0.3 & 3.3 \\
\hline$D P[$ bar $](\mathrm{simp})$ & 0.68 & 0.016 & 1.9 & 0.25 & 6.3 \\
$D P[$ bar $](\mathrm{PDE})$ & 0.66 & $0.01 \pm 0.01 i$ & 4.3 & 0.62 & 6.1 \\
\hline$Q\left[\mathrm{~m}^{3} / \mathrm{s}\right](\mathrm{simp})$ & 0.0196 & - & 1 & 0.09 & 0.28 \\
$Q\left[\mathrm{~m}^{3} / \mathrm{s}\right](\mathrm{PDE})$ & 0.0211 & - & 1 & 0.117 & 0.33 \\
\hline
\end{tabular}

inlet or riser base pressure is the most common industrial approach to stabilizing these systems (Courbot, 1996; Henriot et al., 1999; Havre et al., 2000; Havre and Dalsmo, 2002; Skofteland and Godhavn, 2003). A Bode plot for the process transfer function $G(s)$ from input $u=Z$ to measurement $y=P_{I}$ is shown in figure 6 . The lowfrequency behaviors of the two models are almost identical except for a slightly higher gain around the unstable frequency for the simplified model. The deviations at higher frequencies are caused by time delay and the dampening effects of the fluid dynamics in the feed section of the pipeline that is neglected in the simplified model.

The Bode plot indicates that there might be two potential problems with using the inlet pressure for stabilizing control; the time delay might be too long and the high frequency gain might be too low for effective disturbance rejection. The time delay problem can be omitted by using the pressure at the riser base instead of the inlet pressure. The problems with disturbance rejections at higher frequencies are not directly related to stabilization, but disturbances at higher frequencies are considered to be a problem, these could be suppressed by adding an extra loop in the controller with a measurement that has higher high-frequency gain (Skofteland and Godhavn, 2003).

\subsubsection{Topside flow measurements $W$ and $Q$} The final measurement alternative considered in tables 1 and 2 is the topside volumetric flow $(Q)$. From tables 1 and 2, the system is stabilizable with this measurement as $\min _{K}\|K S\|_{\infty}>1$ for both operating points and models. However, the stationary gain associated with the volumetric

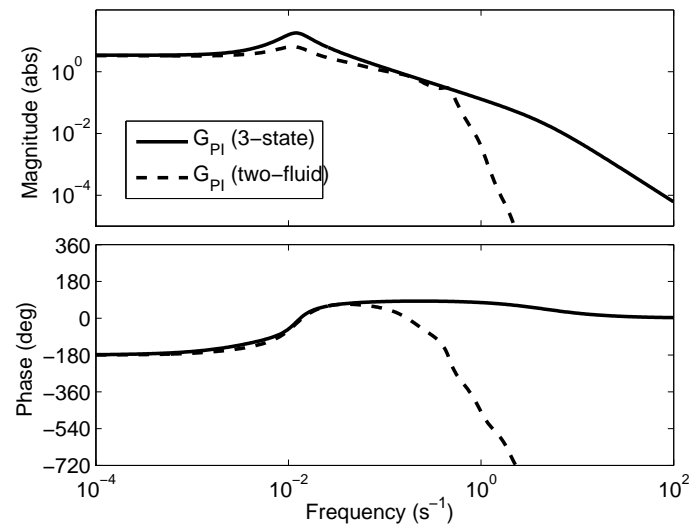

Fig. 6. Bode plot for process model from input $u=Z$ to measurement $y=P_{I}$

flow $Q$ is low. This can also be seen from the Bode plot for the process transfer function $G(s)$ from input $u=Z$ to measurement $y=Q$ in figure 7 . The small low-frequency process gain implies that there will be a problem achieving low-frequency performance (integral action) with this measurement as input to a stabilizing controller. The problem with the lacking low-frequency process gain is that the process might drift from its desired operating point to a operating point which is not stabilizable.

If disturbance rejection at medium to high frequencies is important to keep the input away from saturation and by that keep the system stable, a comparison between figures 6 and 7 indicates that it might be better to use a flow measurement as input to a stabilizing controller. In that case, the pressure measurement could be use in an outer loop in a cascade and by that provide the low- 


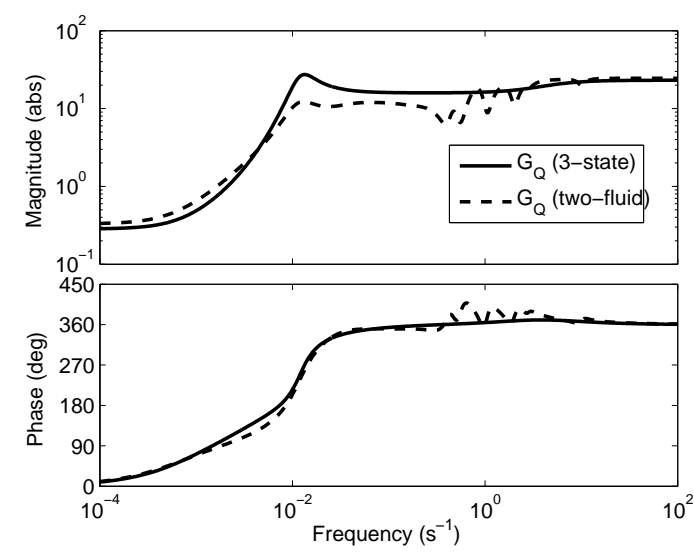

Fig. 7. Bode plot for process model from input $u=Z$ to measurement $y=Q$

frequency performance necessary to keep the system at its desired set point.

\section{CONCLUSION}

A controllability analysis of a pipeline-riser system at riser slugging conditions shows that a good control structure for stabilizing the system is a cascade structure where the flow through the topside choke valve is controlled in the inner loop and the inlet or riser base pressure is controlled in the outer loop. A single pressure loop controlling the inlet or riser base pressure can also a good solution, provided that good disturbance rejection properties at medium to high frequencies are not needed. Single loop flow controllers can also stabilize the system, but small low-frequency gain might cause the operation point to drift.

Although the (linear) system is theoretically stabilizable using pressure drop over the topside choke valve or mixture density at the top of the riser as measurement, it is very sensitive to uncertainty, nonlinearity and model error due to unstable poles and zeros located relatively close to each other in the complex right half plane. Thus, in practice, the pressure drop over the topside choke valve can not be used for stabilizing control.

\section{REFERENCES}

Chen, J. (2000). Logarithmic integrals, interpolation bounds and performance limitations in MIMO feedback systems. IEEE Transactions on Automatic Control AC-45(6), 1098-1115.

Courbot, A. (1996). Prevention of severe slugging in the Dunbar 16" multiphase pipeline. Offshore Technology Conference, May 6-9, Houston, Texas.

Francis, B.A. (1987). Lecture Notes in Control and Information Sciences: A Course in $\mathcal{H}_{\infty}$ Control. Springer-Verlag.
Havre, K. and M. Dalsmo (2002). Active feedback control as a solution to severe slugging. SPE Production and Facilities pp. 138-148. SPE 79252.

Havre, K., K.O. Stornes and H. Stray (2000). Taming slug flow in pipelines. ABB review 4, 55-63.

Hedne, P. and H. Linga (1990). Suppression of terrain slugging with automatic and manual riser choking. Advances in Gas-Liquid Flows pp. 453-469.

Henriot, V., A. Courbot, E. Heintze and L. Moyeux (1999). Simulation of process to control severe slugging: Application to the Dunbar pipeline. SPE Annual Conference and Exhibition in Houston, Texas. SPE 56461.

Hollenberg, J.F., S. de Wolf and W.J. Meiring (1995). A method to supress severe slugging in flow line riser systems. Proc. 7th Int. Conf. on Multiphase Technology Conference.

Kovalev, K., A. Cruickshank and J. Purvis (2003). The slug suppression system in operation. Offshore Europe 2003, Aberdeen, UK. SPE 84947.

Schmidt, Z., J.P. Brill and H.D. Beggs (1979). Choking can eliminate severe slugging. Oil and Gas Journal pp. 230-238.

Skofteland, G. and J.-M. Godhavn (2003). Suppression of slugs in multiphase flow lines by active use of topside choke - field experience and experimental results. In: Proc. of MultiPhase '03, San Remo, Italy, 11-13 June 2003.

Skogestad, S. and I. Postlethwaite (1996). Multivariable feedback control. John Wiley \& sons.

Storkaas, E., S. Skogestad and J.-M. Godhavn (2003). A low-dimensional model of severe slugging for controller design and analysis. In: Proc. of MultiPhase '03, San Remo, Italy, 1113 June 2003.

Storkaas, E., V. Alstad and S. Skogestad (2001). Stabilization of desired flow regimes in pipelines. AIChE Annual Meeting 2001, Reno, Nevada. Paper 287d.

Taitel, Y. (1986). Stability of severe slugging. Int. J. Multiphase Flow 12(2), 203-217.

Thompson, J.M.T. and H.B. Stewart (1986). Nonlinear dynamics and chaos. John Wiley \& sons.

Youla, D. C., H.A. Jabr and C.N. Lu (1974). Single-loop feedback stabilization of linear multivariable dynamical plants. Automatica 10, 159-173. 